\title{
Fabrication of refraction and diffraction glass lenses by using hot embossing process
}

\author{
R. Kasztelanic, ${ }^{* 1}$ I. Kujawa, ${ }^{2}$ R. Stępień, ${ }^{2}$ A.J. Waddie, ${ }^{3}$ M.R. Taghizadeh, ${ }^{3}$ and R. Buczyński ${ }^{1,2}$ \\ ${ }^{1}$ Faculty of Physics, Warsaw University, Pasteura 7, 02-093 Warszawa, \\ ${ }^{2}$ Glass Laboratory, Institute of Electronic Materials Technology, Wólczyńska 133, 01-919 Warszawa, \\ ${ }^{3}$ School of Engineering and Physical Sciences, Heriot-Watt University, Edinburgh EH14 4AS, Scotland, UK
}

Received October 14, 2013; accepted December 17, 2013; published December 31, 2013

\begin{abstract}
The paper focuses on the results of fabrication of diffraction and refraction lenses with the use of hot embossing process from multi-component glasses. Hot embossing is an attractive alternative to other micro-replication methods, since its cost effectiveness makes it suitable for mass-fabrication of wide range of elements. Multi-component glasses names soft glasses are transparent from visible to near and mid infrared spectral region.
\end{abstract}

There are several methods used for large volume production of optical refractive elements. Most of these replication methods, such as injection molding and hot embossing, are well suited for polymers [1-2]. However, they are not usually applied to the processing of glass, since high temperature and crystallization are an issue.

The hot embossing (HE) process [3-4] used for the manufacturing of glass optical elements can be divided into three main phases (Fig. 1). In the first, initial phase, the material and the stamps are heated to the desired temperature. The second phase of the embossing process, i.e. pressing, takes place after the optimal temperature of the material has been reached. At this stage, the material is subject to pressure from the stamp, which transfers the pattern image. The parameters which are significant for the process include: an accurately stabilized temperature, the pressure force and dynamics, time of stopping the stamp and the rate of its retraction from the surface. The material chosen for the stamp, the size of the heating chamber and moment of the process chamber area unsealing, also affect the HE process. During the third phase, the element can be cooled and removed from the stamping device. Care has to be taken, that the fabricated element does not suffer mechanical damage or crystallization at this final phase.

As noted above, important factors that affect the quality of the received elements are glass and the material of the stamp. For the initial experiments we chose glasses which were potentially suitable for the visible spectrum and mid-infrared (Fig. 2). These were glasses composed of three to six oxides in varying proportions: $\mathrm{SiO}_{2}, \mathrm{PbO}$, $\mathrm{Bi}_{2} \mathrm{O}_{3}, \mathrm{Ga}_{2} \mathrm{O}_{3}, \mathrm{CdO}, \mathrm{B}_{2} \mathrm{O}_{3}, \mathrm{ZnO}, \mathrm{Tl}_{2} \mathrm{O}, \mathrm{GeO}_{2}, \mathrm{WO}_{3}, \mathrm{Na}_{2} \mathrm{O}$, $\mathrm{TeO}_{2}$ and $\mathrm{Nb}_{2} \mathrm{O}_{5}$. As a glass selection criteria we used

\footnotetext{
*E-mail: kasztel@igf.fuw.edu.pl
}

transmission properties in infrared region, adhesion between glass and the mold insert, as well as the viscosity characteristic and resistance to crystallization. In addition we took into account also cost and availability. We established that glasses PBG08 and TWPN/I/6 are characterized by the best resistance to crystallization [5].

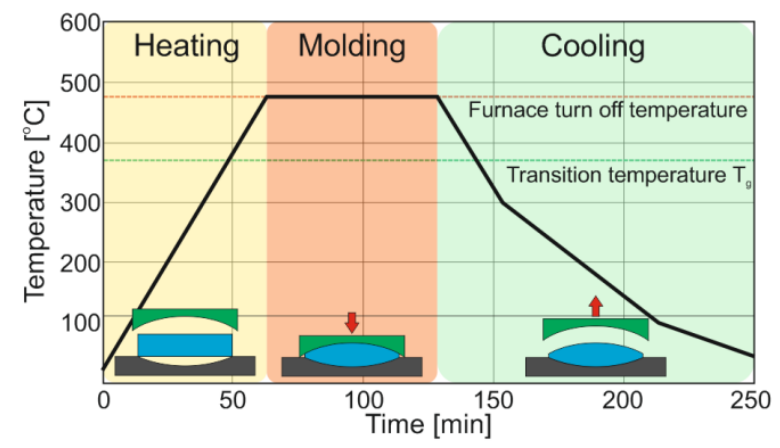

Fig. 1. Scheme of the hot embossing process.

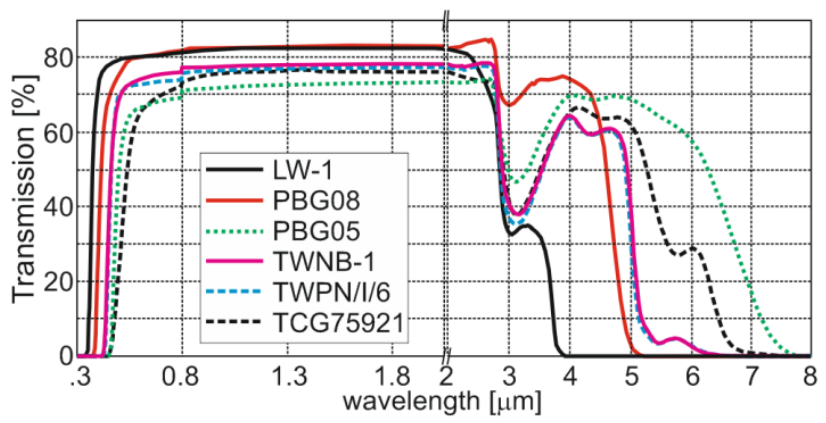

Fig. 2. Spectral transmission of the glasses for a $2 \mathrm{~mm}$ sample.

Another significant technological issue is the adhesion of the glasses to the mold surface during thermal treatment. What is essential for this process is not only the viscosity, but also the surface tension and the wetting between the glass and the material of the stamp during embossing. We studied the interaction between glasses and various stamp material, as well assist dependence on temperature, in order to estimate their adhesion. Glass adhesion was tested for various metals and for nonmetallic materials. Finally, for stamp fabrication, 
taking into account the parameters of glass adhesion to molds made of different materials and the ability to manufacture appropriate forms, further work was carried out with the use of molds made of fused silica glass. The molds were fabricated with the use of ion etching technology.

The device setup for $\mathrm{HE}$, designed and prepared at Institute of Electronic Materials Technology, is shown in Fig. 3. The device comprises a furnace (1) with a controller attached to the steel chassis (2). Inside the chamber of furnace, a glass substrate (3) is placed on the lower holder of the stencil (4). The temperature is measured with a thermocouple (5). Above the furnace there is an air powered cylinder (6) controlled by an electro-valve. The base of piston and the pedestal are water-cooled (7).
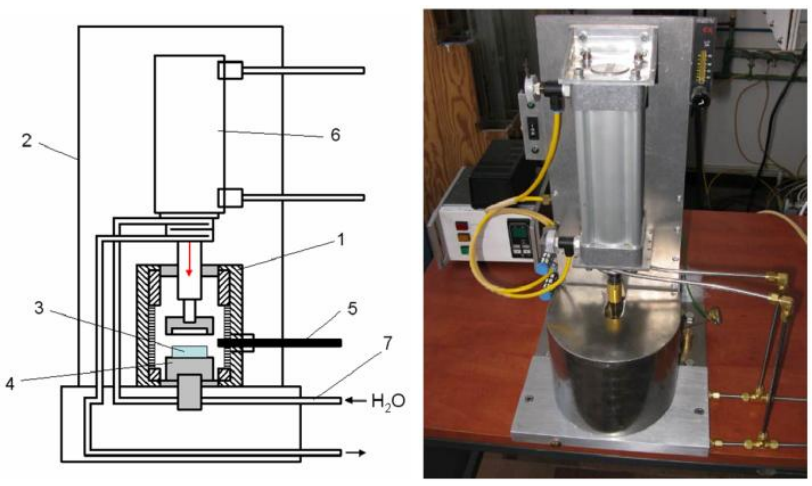

Fig. 3. Schematic drawing of HE setup and developed device.

A series of test processes was performed using the described apparatus. The parameters examined included: the actual working temperature, heating and cooling rates, the speed of piston movement and force pressure. The tests yielded a set of operational parameters suitable for prototyping the actual optical elements for individual glasses and stamps.

In the case of diffractive elements a single mold consisted of an array of $64(8 \times 8)$ of Fresnel lenses, each with a diameter of $750 \mu \mathrm{m}$ (Fig. 4a). The optimal parameters established for molding from the PBG08 glass were: pressure of $32.5 \mathrm{bar}$, temperature of the molding $\mathrm{T}_{\mathrm{s}}=518^{\circ} \mathrm{C}$, forming time $\mathrm{t}=120 \mathrm{~min}$ [6]. Importantly, when cooling the fabricated element shrinking is negligibly small, so it does not change the optical properties. The example result of the replication is presented in Fig. 4b.

In the case of refractive lenses, we tested the development of different shapes of lenses: plano-concave, plano-convex and biconvex lenses with a diameter of 3 to $10 \mathrm{~mm}$ and thickness up to $2 \mathrm{~mm}$. Another series of test processes was carried out to optimize its parameters, in particular the working temperature, imprint force, speed of heating and cooling. The optimum embossing temperature was established at $544^{\circ} \mathrm{C}$ for the PBG08 glass and at $468^{\circ} \mathrm{C}$ for the TWPN/I/6 glass. The total process time was $380 \mathrm{~min}$ for the PBG08 and $210 \mathrm{~min}$ for the TWPN/I/6 glass. The constant imprint force of $1.0 \mathrm{~N}$ was used for the PBG08 glass and $1.22 \mathrm{~N}$ for the TWPN/I/6 glass (Fig. 5a) [7]. The wrong choice of the temperature or length of the processing cycle results in the formation of air bubbles or crystallization of glass (Fig. 5b). On the other hand, the proper selection of the process parameters allows for easy separation of the fabricated element from the stamp.

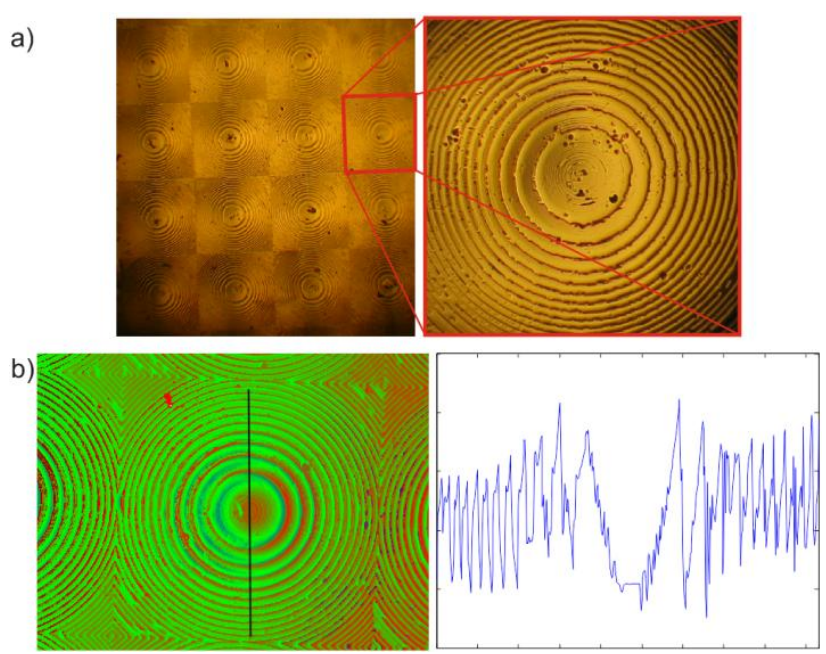

Fig. 4. Image of the Fresnel lens: a) stamp, b) molded element.
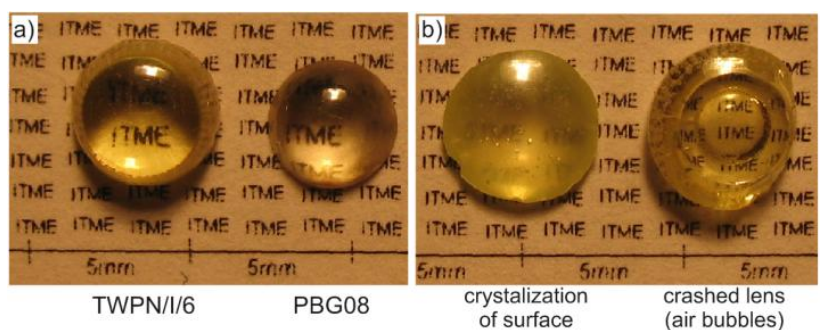

Fig. 5. Lenses: a) imaging properties of the developed biconvex mini lenses made of TWPN/I/6 and PBG08 glasses, b) lenses are made with the wrong choice of parameters.

The profilometer measurements were used to study the surface curvature and the quality of the optical surface of molded lenses (Fig. 6). The results obtained for various stamps show identical curvature of the mold and the replicated lenses but the quality of the obtained surface is worse than the mold. Some cracks occurring on the surface result from a mismatch of the expansion coefficients of the molded soft glass and fused silica of the mold. The observed lack of spherical symmetry for the concave lens may be the result of errors of adjustment of both parts of the stamp.

At this initial stage it is difficult to estimate the repeatability of the manufactured items because emphasis was placed on the selection of the suitable process parameters. 


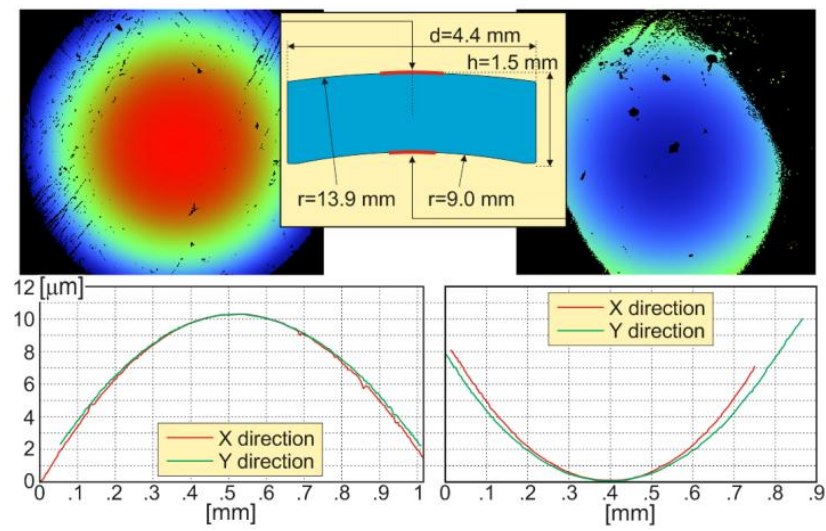

Fig. 6. The measurement results and profiles from light profilometer for concave-convex lenses made of TWPN/I/6 glass.

The optical and imaging properties of the fabricated elements were verified with various methods. We measured the focal length of lenses and compared the results with the theoretical values obtained on the basis of the refractive index, thickness and measured surface curvature of the lens. The results obtained for most of the lenses show that the difference between the expected and the measured focal length was less than $1 \%$.

The resolution of the molded lenses was verified with standard test targets resolution 1951 USAF using the method based on the modulation (contrast) transfer function (MTF). The MTF is commonly used to measure the quality of imaging lenses. It represents how accurately the tested lens reproduces details of an imaged object.

The MTF defined as:

$$
M T F=\frac{\text { Image modulation }}{\text { object modulation }}
$$

was obtained by measuring the contrast (modulation) of a series of black and white images where number of lines per millimeter is systematically increased (Fig. 7). The contrast for a given frequency (line pairs per millimeter) $C_{f}$ is defined as:

$$
C_{f}=\frac{I_{\max }-I_{\min }}{I_{\max }+I_{\min }}
$$

where $I_{\min }$ and $I_{\max }$ denotes the minimum intensity (pixel value) and maximum intensity, respectively for each resolution test. For measurements, we used linear CCD camera with gamma correction $\gamma=1$. The MTF diagram (Fig. 7) was finally used to determine the cutoff frequency (resolution) defined at $50 \%$ of MTF. The final result for the molded lens at the wavelength of $1300 \mathrm{~nm}$ is $37 \mathrm{p} / \mathrm{mm}$ (line pairs per millimeter). Due to the light source available we did not measure chromatic aberration.

The fabricated mini lenses were also used for the construction of microscope objectives. In the microscope setup the developed microscope objective was combined with a standard ocular lens eyepiece of the magnification $10 \times$. For this reason the microscope setup was tested only in the near infrared range at $1300 \mathrm{~nm}$. We experimentally verified the resolution to be $501 \mathrm{p} / \mathrm{mm}$.

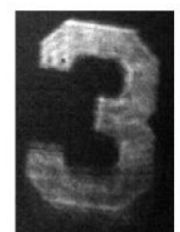

$1.26 \mathrm{Ip} / \mathrm{mm}$

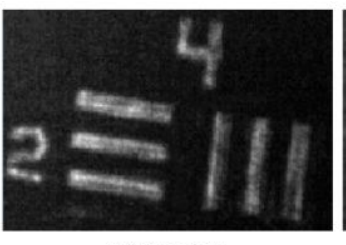

$17.95 \mathrm{Ip} / \mathrm{mm}$

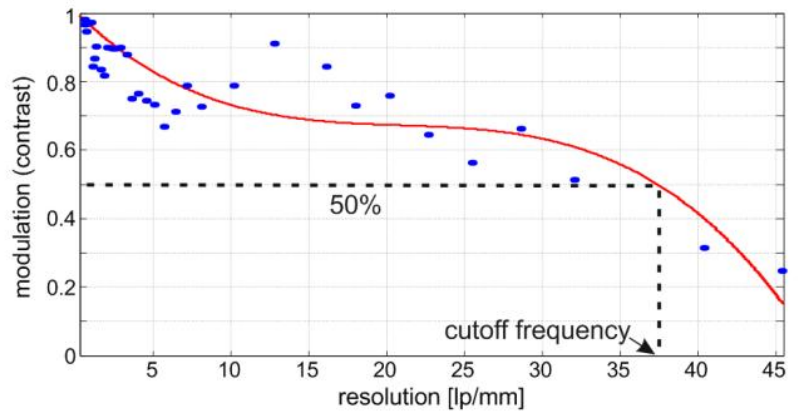

Fig. 7. Imaging of the test targets with various resolutions for refractive lens and measured modulation transfer function (MTF) for this lens.

In conclusions, we have successfully developed thermally and mechanically stable glass with broadband transmission from visible till mid infrared up to 6 micrometers. We presented the major steps involved in the fabrication of refractive and diffractive lenses in the hot embossing process. We obtained good image properties of the developed lenses and we proved the ability of the lens to focus light in the visible and in the mid-infrared spectral range. Finally, we showed that the fabricated lenses can be used for imaging, and may be used as a part of larger optical devices, such as a microscope.

This research was carried out with the financial support from the Polish Ministry of Science and Higher Education research grant N507 431339 and the project operated within the Foundation for Polish Science Team Programme co-financed by the European Regional Development Fund, Operational Program Innovative Economy 2007-2013.

\section{References}

[1] H. Becker, U. Heim, Sensors and Actuators 83, 130 (2000).

[2] M. Worgull, Hot Embossing, Theory and Technology of Microreplication, (William Andrew, 2009).

[3] M. Heckele, W.K. Schomburg, J. Micromech. Microeng. 14, R1 (2004).

[4] C.T. Pan, T.T. Wu, M.F. Chen, Y.C. Chang, C.J. Lee, J.C. Huang, Sensors and Actuators A 141, 422 (2008).

[5] I. Kujawa, R. Kasztelanic, R. Stępień, M. Klimczak, J. Cimek, A.J. Waddie, M.R. Taghizadeh, R. Buczyński, Opt. \& Laser Techn. 55, 11 (2014).

[6] R. Kasztelanic, I. Kujawa, R. Stępień, K. Haraśny, D. Pysz, R. Buczyński, Infrared Phys. \& Techn. 60, 1 (2013).

[7] R. Kasztelanic, I. Kujawa, R. Stępień, K. Haraśny, D. Pysz, R. Buczyński, Infrared Phys. Techn. - in press. 